\title{
Fetal origin of allergic asthma: insights on mechanistic cues and therapeutic targets arising from a mouse model of prenatal stress challenge
}

\author{
Christian A Bruenahl', Maike Pincus², Emilia Solano ${ }^{1}$, Evelin Hagen ${ }^{1,2}$, Astrid Friebe ${ }^{1}$, Russ Ellis ${ }^{1}$, Mark Inman ${ }^{1}$, \\ Petra C Arck ${ }^{*}$ \\ From AllerGen NCE Inc.'s Fifth Annual Research Conference: Innovation from Cell to Society \\ Québec City, QC, Canada. 7-9 February 2010
}

\section{Background}

Prenatal stress challenge is a pivotal environmental factor which has been proposed to increase the vulnerability of offspring to develop chronic immune diseases in later life. We analyze the effect of prenatal exposure to stress during late gestation in mice.

\section{Materials and methods}

$\mathrm{BALB} / \mathrm{c}$ mice were exposed to sound stress during late gestation. Maternal serum and placentas were analyzed. Fetal development was scored. Allergic asthma was induced in the offspring by using an ovalbumin protocol. We analyzed immune cells in lungs, bronchioalveolar fluid (BAL) and lung-draining lymph nodes as well as cytokine concentrations in the BAL. Further, stress-challenged pregnant females were treated with a progesterone derivative, followed by fetal analyses and evaluation of the vulnerability towards asthma in the offspring.

\section{Results}

Stress challenge resulted in decreased serum levels of maternal progesterone and testosterone, and increased serum levels of estradiol associated with placental endocrine dysfunction, such as low expression of proliferin. Fetal development was impaired upon stress challenge, especially in females. Prenatally stressed female adult offspring revealed an increased susceptibility toward asthma, mirrored by an increased airway response, influx of inflammatory cells and increased $\mathrm{T}$ helper 2 cytokines in the BAL. Further, we observed decreased frequencies of regulatory $\mathrm{T}$ cells $\left(\mathrm{CD}^{+} \mathrm{CD}^{+} \mathrm{CD} 25\right.$ ${ }^{+}$foxP $3^{+}$. Progesterone supplementation abrogated the impaired intrauterine development as well as the susceptibility toward asthma.

\section{Conclusions}

Our study revealed that prenatal stress severely interferes with the intrauterine development, resulting in offspring with an increased vulnerability toward asthma-like symptoms. Supplementation of progesterone during stress-challenged pregnancies abrogates gender-dependently the increased susceptibility toward asthma.

\section{Author details}

'McMaster University, St. Joseph's Healthcare, Hamilton, Ontario, Canada.

${ }^{2}$ Charité, University Medicine Berlin, Germany.

Published: 26 November 2010

doi:10.1186/1710-1492-6-S3-P13

Cite this article as: Bruenahl et al:: Fetal origin of allergic asthma: insights on mechanistic cues and therapeutic targets arising from a mouse model of prenatal stress challenge. Allergy, Asthma \& Clinical Immunology 2010 6(Suppl 3):P13. 